\title{
Religiosidade e Bem-Estar Psicológico de Acadêmicos de Psicologia
}

\author{
Timoteo Madaleno Vieira ${ }^{a *}$, Daniela Sacramento Zanini ${ }^{b}$ \& Alexandre de Paula Amorim ${ }^{b}$ \\ ${ }^{a}$ Instituto Federal de Educação, Ciência e Tecnologia de Goiás, Goiânia, Brasil;

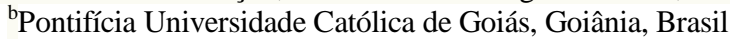

\begin{abstract}
RESUMO
O presente estudo avaliou a atitude religiosa, a interação entre religiosidade e formação em psicologia, e o bem-estar psicológico e físico de 168 estudantes de psicologia. Encontraram-se correlações positivas entre a religiosidade e o bem-estar; posicionamentos negativos de professores frente à religiosidade; bem como conflitos entre crenças religiosas dos alunos e algumas abordagem de psicologia. Considerou-se a formação em psicologia como possível evento estressor diante do qual o conflito entre a religiosidade e a formação em psicologia pode se tornar um fator de risco. O bem-estar positivo foi assumido como evidência de saúde. Discute-se o papel dos professores de psicologia na promoção da saúde e propõe-se que sejam desenvolvidas ações de prevenção da saúde dos alunos de psicologia.
\end{abstract}

Palavras-chave: fatores de risco; fatores de proteção; religiosidade; bem-estar.

\begin{abstract}
Religiosity and Psychological Well-Being of Psychology Students

The present study assessed the religious attitude, the interaction between religiosity and psychology education, and the physical and psychological wellbeing of 168 psychology students. Positive correlations between religiosity and wellbeing; negative positioning of psychology teachers facing the religiosity; and conflict between the religious beliefs of the students and some psychology approaches were found. The education in psychology was considered as a possible stressful event that could make the conflict between religiosity and education in psychology became a risk factor. The positive wellbeing was considered as evidence of health. It is discussed the role of the psychology teachers in promoting health and it is proposed that preventive actions were developed for the health of psychology students.
\end{abstract}

Keywords: risk factors; protective factors; religiosity; wellness.

O início da vida acadêmica ocorre, para muitos, em um momento crítico da vida, envolvendo incertezas quanto ao futuro e mudanças nas relações sociais e na autopercepção (Cerchiari, 2004). Alguns estudantes podem enfrentar tal processo de modo mais saudável, devido a recursos psicológicos e sociais que os tornam mais preparados para os desafios envolvidos na formação acadêmica (Fontes, 2010; Pesce, Assis, Santos, \& Oliveira, 2004; Pinheiro, 2004). Entretanto, é necessário considerar que esses recursos podem ser afetados, positivamente ou negativamente, durante os anos de formação. No processo de formação parte das bases cognitivas que orientam as interpretações da realidade pode ser confrontada pelo posicionamento acadêmico-científico frente a questões relacionadas à espiritualidade/religiosidade, valores pessoais e familiares, etc.

Os objetivos do presente estudo foram: Verificar as relações entre religiosidade e bem-estar psicológico no contexto acadêmico; verificar a percepção de alunos de psicologia acerca do conflito entre a religiosidade e a formação em psicologia; verificar, a partir de relatos de alunos, como os professores de psicologia se posicionam frente às crenças religiosas dos estudantes. 
Para isto apresentar-se-ão os conceitos de resiliência, fatores de risco e proteção e bem-estar psicológico. Serão avaliados a formação em psicologia e seu conflito com a religiosidade do estudante como possíveis eventos estressores e fatores de risco.

\section{Fatores de risco e de proteção da saúde}

Desde o nascimento, os indivíduos são expostos a desafios impostos pelo ambiente natural, social, cultural e por eventos específicos de vida. Alguns desses desafios são considerados como estressores, por exigirem dos indivíduos novas adaptações. A psicologia vem se interessando em compreender as diferenças individuais observadas no enfrentamento desses desafios (Lazarus \& Folkman, 1984; Snyder \& Lopez, 2009). Enquanto alguns indivíduos apresentam grande capacidade de superação e desenvolvimento a partir das adversidades, outros se mostram mais vulneráveis (Pinheiro, 2004). Essa capacidade de enfrentar situações complexas e ameaçadoras e de desenvolver-se a partir disso tem sido chamada por alguns psicólogos de resiliência (Paula Junior \& Zanini, 2011).

Para Pesce et al. (2004), a resiliência envolve um conjunto de processos internos e sociais que podem possibilitar aos indivíduos desenvolverem-se de modo saudável mesmo em ambientes não saudáveis. Fontes (2010) destaca a plasticidade, diante de um mundo dinâmico, como a principal característica da resiliência. Esta perspectiva considera a resiliência como processo adaptativo constante e fundamental para o desenvolvimento, o que sugere que indivíduos que conseguem reunir mais recursos cognitivos e psicossociais podem se tornar mais resilientes ao longo da vida.

Estudos sobre resiliência têm apontado que existem fatores de risco ou vulnerabilidade que podem afetar de modo negativo a adaptação do indivíduo. Do mesmo modo, são apontados fatores considerados protetores por contribuírem para tornar as pessoas menos vulneráveis diante de desafios ou adversidades (Fontes, 2010; Pesce et al., 2004; Pinheiro, 2004). Jessor, Van Den Bos, Vanderryn, Costa e Turbin (1995) consideram que fatores de risco são condições ou variáveis que tornam mais prováveis resultados negativos ou indesejáveis, bem como comportamentos que ameaçam a saúde, o bem-estar ou o desempenho em situações sociais. Os autores, entretanto, ressaltam a necessidade de cuidados na definição de cada fator como sendo de risco ou de proteção. Deve-se compreender que em certos ambientes um fator pode ser protetor por contribuir com a diminuição de riscos específicos, mas em outros ambientes este mesmo fator pode ser considerado de risco. A facilidade de um adolescente para interagir socialmente e formar novas amizades, por exemplo, pode ser um importante fator protetor em vários ambientes, mas pode ser um fator de risco em um bairro onde há um grande número de adolescentes usuários de drogas (Jessor et al., 1995). Nesse sentido, ressalta-se a complexidade dos processos que podem tornar as pessoas mais vulneráveis ao adoecimento ou mesmo resilientes.

\section{Religiosidade, espiritualidade e bem-estar psicológico}

Segundo Gastaud et al. (2006) o conceito de espiritualidade envolve um elemento vertical, religioso (um sentimento de harmonia com um ser superior), e também um elemento horizontal, existencial, que diz respeito à compreensão de um propósito para a existência humana. O segundo elemento, horizontal, não implica necessariamente referências ao religioso. A religiosidade é mais específica: "Religiosidade é a extensão na qual um indivíduo acredita, segue e pratica uma religião" (Gastaud et al., 2006, p.13). Embora estes conceitos estejam entrelaçados, o presente trabalho centra-se especificamente no estudo da religiosidade, objetivada na atitude religiosa de estudantes de psicologia. A atitude religiosa é medida pelo envolvimento dos indivíduos em práticas religiosas como orações, leituras de material religioso, adoção de crenças definidas a partir de uma tradição religiosa específica, participação em cultos e outras reuniões religiosas, etc. Essa opção se justifica pela pressuposição de que pessoas que apresentam uma atitude religiosa mais clara, com um envolvimento religioso significativo e bem definido, podem adotar as crenças religiosas como referenciais cognitivos importantes para as suas interações. Essas crenças podem ser orientadoras e definir posicionamentos cognitivos e comportamentais do indivíduo frente a situações de risco e eventos estressores. Além disso, ao serem orientadoras, podem promover segurança e senso de sentido para a existência.

Apesar do crescimento do interesse científico pela religiosidade e a espiritualidade, ainda é possível encontrar uma tendência por parte dos psicólogos e psiquiatras de vê-las de modo pejorativo, relacionando-as com neuroses, atitudes irracionais, comportamento supersticioso ou infantilidade (Fleck, Borges, Bolognesi, \& Rocha, 2003; Freud, 1927/1969; Gastaud et al., 2006; Koenig, 2007; Skinner, 1953). A origem desta posição é histórica na psicologia. Para Freud, a religião representa para a vida social o que a neurose obsessiva representa para o indivíduo (1907/1969). 
Um ponto importante da visão freudiana da religião trata especificamente da concepção judeu-cristã de Deus e suas influências sobre a cultura ocidental. A culpa imposta pelo Deus-Pai ambivalente (que é ao mesmo tempo ameaçador e um protetor idealizado) produziria um modo infantilizado de interação com a realidade. A infantilidade se dá na construção de uma relação com a realidade marcada pela culpa e pelos desejos e não pela razão. A proposta de Freud é de uma sociedade construída através da educação puramente científica das crianças, sem a interferência da religião, o que permitira o desenvolvimento de uma sociedade emancipada e amadurecida (Freud, 1927/1969).

Mais recentemente, Ellis (1988) e Watters (1992) (citados por Koenig, (2007), também enfatizaram o caráter irracional das crenças religiosas e seus efeitos prejudiciais ao indivíduo. Tais posicionamentos em relação à religião ainda exercem influência sobre as atitudes de muitos profissionais de psicologia (incluindo professores), de acordo com Gastaud et al. (2006).

Estudos recentes, entretanto, têm mostrado que as crenças religiosas podem ter efeito protetor da saúde humana, especialmente quando fomentam a esperança e atitudes positivas diante da vida, produzindo maior capacidade de enfrentamento de situações negativas (Costa et al., 2008; Panzini \& Bandeira, 2005; Panzini, 2004). A espiritualidade/religiosidade tem se mostrado como um fator protetor para suicídio, comportamentos de risco, insatisfação marital, sofrimento psicológico, e etc. (Fleck, Borges, Bolognesi, \& Rocha, 2003; Gastaud et al., 2006; Panzini, Rocha, Bandeira, \& Fleck, 2007; Panzini, 2004; Pargament, 1997; Seybold \& Hill, 2001; Stroppa \& Moreira-Almeida, 2008). Tais evidências reforçam a hipótese de que as crenças religiosas dos estudantes de psicologia são relevantes para a manutenção do seu bem-estar e da sua saúde ao longo de suas vidas. O bem-estar, como medida subjetiva, é aqui assumido como um indicador de saúde, sem desconsiderar a complexidade de ambos os conceitos.

Sob a influência do crescimento da Psicologia Positiva (Passareli \& Silva, 2007), psicólogos e outros profissionais da saúde vêm se interessando pelo estudo das potencialidades humanas. O bem-estar subjetivo é um dos interesses da Psicologia Positiva, estando relacionado a tais potencialidades. O termo "subjetivo" significa que o bem-estar depende da autopercepção do indivíduo e o relato que este apresenta. De acordo com Passareli e Silva (2007), este conceito diz respeito ao modo como as pessoas veem a si mesmas e aos outros, bem como ao prazer em vivenciar as experiências cotidianas e os relacionamentos.
Para Diener (1984) o bem-estar subjetivo tem três componentes: satisfação com a vida, afeto positivo e afeto negativo. Tais componentes são considerados como cognitivos, já que envolvem crenças autoavaliadoras da vida (atitudes). As interações entre tais componentes e entre os diversos domínios do que o autor denomina "satisfação com a vida" (por exemplo: satisfação com a saúde) é bastante complexa e tem ocupado autores interessados em discuti-las (Schimmack, 2008). Traços de personalidade também têm sido considerados importantes preditores do bem-estar subjetivo (Steel, Schmidt, \& Schultz, 2008).

Apesar do termo "bem-estar subjetivo" ser bastante utilizado, alguns autores preferem o uso do conceito "bem-estar psicológico" (Siqueira \& Padovam, 2008). Para estes, o conceito de bem-estar psicológico possui uma sustentação conceitual mais sólida, por ser fundamentado por componentes bem definidos, como: autoaceitação, relacionamento positivo com as pessoas, autonomia, domínio do ambiente, propósito de vida, crescimento pessoal. O presente trabalho adota o conceito de bem-estar psicológico, que fundamenta teoricamente o Questionário de Bem-Estar Psicológico e Físico, aqui utilizado (Oliveira, Torres, \& Albuquerque, 2009).

No contexto brasileiro, que é predominantemente religioso, parece haver uma forte tendência a interpretações da realidade mediadas por crenças religiosas (Pierucci, 2004). Segundo o modelo cognitivo de Aaron Beck, as crenças são modos de interpretação da realidade através dos quais os indivíduos organizam o pensamento e atribuem significado às experiências (Beck, 1997; Falcone, 2001). A saúde, em seu sentido integral (sem a separação mente-corpo ou afins), parece estar relacionada às interpretações que as pessoas fazem da realidade (crenças). Tomando o modelo cognitivo como referência, o presente trabalho compreende as crenças religiosas como sendo afirmações que orientam interpretações da realidade e são orientadas por pressupostos de origem religiosa. Alguns exemplos desses pressupostos são: a afirmação da existência de Deus, atribuições de causalidade a eventos reais por parte de intervenção divina, ou seja, utilização de explicações metafísico-transcendentes para compreensão de fenômenos da realidade, existência de vida após a morte, etc. Considerando que os estudantes de psicologia brasileiros fazem parte de uma população religiosa, supõe-se que as crenças religiosas desempenham papel importante para o funcionamento cognitivo dos mesmos e podem compor um fator de proteção da sua saúde. 


\section{DELIMITAÇÕES E OBJETIVOS}

O presente trabalho apresenta a possibilidade de que a formação em psicologia seja um evento estressor para o adoecimento psicológico de estudantes. O conhecimento científico orientado por concepções de ciência originadas da modernidade e ainda predominantes na academia, pode questionar o sistema de crenças dos estudantes religiosos, exigindo uma reestruturação cognitiva (Giddens, 1991). Cavestro e Rocha (2006) estimam que $15 \%$ a $25 \%$ dos estudantes universitários apresentam algum tipo de transtorno psicológico durante a sua formação acadêmica. $\mathrm{O}$ curso de psicologia parece estar entre os que apresentam maiores índices de adoecimento psicológico de estudantes, como mostram alguns trabalhos recentes (e.g., Bouteyre, Maurel, \& Bernaud, 2007; Sakae, Parão, \& Jornada, 2010; Teixeira, Fonseca, \& Maximo, 2002; Yoon \& Lau, 2008).

Apesar dos autores supracitados focarem especificamente o adoecimento psicológico, é importante considerá-lo dentro de um contexto mais amplo. Para a Organização Mundial de Saúde (OMS) a saúde é definida como sendo o bem-estar biológico, psicológico e social (Fleck et al., 1999; Jarros et al., 2004). O conceito de saúde adotado pela OMS valoriza a própria percepção que o indivíduo tem acerca de si mesmo, dos outros e da vida. A autopercepção positiva do bem-estar, nesta perspectiva, pode ser considerada como evidência de saúde.

O presente trabalho avalia o papel da religiosidade em termos de proteção e risco. Considerando a concepção dinâmica de Jessor et al. (1995) acerca dos fatores de risco e proteção, é importante situar que as crenças religiosas, apesar de serem protetoras (quando fomentam a esperança e oferecerem suporte no enfrentamento de eventos negativos de vida), podem também estar relacionadas a riscos no contexto acadêmico. O conflito entre as crenças religiosas e o conhecimento científico específico de algumas abordagens da psicologia pode potencializar conflitos internos, afetando negativamente a saúde. Vale considerar que os principais mediadores entre os estudantes e a formação acadêmico-científica são os professores. O modo como tal mediação é realizada também é objeto de estudo no presente trabalho.

O principal objetivo do presente trabalho foi realizar uma análise das relações entre a religiosidade dos estudantes de psicologia e o seu bem-estar (considerado como um indicador de saúde) especificamente no contexto da formação acadêmica.
Mais especificamente o presente estudo pretende verificar as relações entre bem-estar e religiosidade; verificar se há conflito entre o curso de psicologia e as crenças religiosas dos estudantes, e; avaliar a percepção dos alunos das atitudes dos professores frente as suas crenças.

\section{MÉTODO}

\section{Participantes}

Participaram do estudo 168 estudantes de psicologia, de ambos os sexos, selecionados segundo critério de conveniência em uma universidade filantrópica da cidade de Goiânia. Os estudantes foram contatados de acordo com o período que cursavam e posteriormente, para fins de análise, foram agrupados em três grupos: início do curso (primeiro, segundo e terceiro períodos), meio do curso (quinto, sexto e sétimo períodos) e final do curso (oitavo, nono e décimo períodos).

\section{Instrumentos}

Foram aplicados três instrumentos, formando um caderno único de coleta, com tempo total de respostas estimado em aproximadamente 30 minutos. Os instrumentos são:

1. Questionário de Avaliação de Atitude Religiosa (QAAR) - Instrumento elaborado por Aquino et al. (2009), com o objetivo de avaliar a religiosidade e a fé dos participantes. É composto por 16 itens distribuídos de acordo com os componentes da atitude - afeto, cognição e comportamento - avaliando as atitudes do participante em relação a cada uma delas. Cada item avalia o grau de frequência das práticas e sentimentos religiosos mencionados, de acordo com uma escala do tipo likert (com graduações numéricas de 1 a 5) que varia de nunca a sempre. O instrumento também avalia a religiosidade em um único fator (atitude religiosa total), cuja consistência interna foi verificada, apresentando Alfa de Cronbach de 0,91 no estudo original.

2. Questionário de Avaliação da Interação Religiosidade-Formação em Psicologia (QARPSI) - Desenvolvido especificamente para esta pesquisa, solicita dados demográficos: sexo, idade, renda familiar e filiação religiosa. O questionário também é composto por nove perguntas fechadas, a serem respondidas em uma escala do tipo likert de cinco pontos, que visa obter as seguintes informações: afirmação do quão religioso o participante se considera, interações entre as crenças religiosas do estudante e o conteúdo do curso de psicologia (aulas e durante estudo fora das 
salas de aula), apoio familiar à opção do estudante pelo curso de psicologia, opiniões sobre as posturas dos professores de psicologia acerca da religiosidade, apoio da comunidade religiosa à opção do estudante pelo curso de psicologia.

3. Questionário de Bem-Estar Psicológico e Físico - Utilizado por Oliveira, Torres e Albuquerque (2009). Através de uma escala do tipo likert de sete pontos, com 32 itens, o instrumento avalia o bem-estar físico e psicológico. Análises anteriores mostraram a existência de um fator único, que foi transformado em um único índice de bem-estar físico e psicológico e demonstrou consistência interna com Alfa de Cronbach variando entre 0,90 e 0,96 , nos diferentes estudos (Oliveira \& Torres, 2005; Rabelo \& Torres, 2005, citados por Oliveira et al., 2009).

\section{Procedimentos}

A pesquisa foi realizada tendo como base a resolução 196/1996 do Conselho Nacional de Saúde (CNS), bem como os procedimentos éticos sugeridos pela American Psychological Association (A.P.A.).

A coleta de dados (aplicação dos instrumentos apresentados na seção anterior) foi realizada em salas de aula do curso de psicologia e por meio da secretaria e dos supervisores de estágio, com o conhecimento da diretoria do departamento de psicologia e da coordenação do centro de estágio em psicologia da instituição.

Para coleta em sala de aula, foi solicitada a autorização de cada professor responsável pela aula no dia e horário da coleta. Antes da aplicação dos instrumentos, todos os participantes foram convidados a ler o Termo de Consentimento Livre e Esclarecido. O termo informa a natureza e os objetivos da pesquisa e solicita a formalização da concordância de participar voluntariamente, bem como a possibilidade de retirada do consentimento a qualquer momento do processo sem qualquer ônus. Os que concordaram foram convidados a assinar o Termo de Consentimento Livre e Esclarecido.

No centro de estágio, os estagiários (estudantes do final do curso) foram convidados a participar (o convite foi feito presencialmente e por e-mail, mas todos os questionários foram aplicados presencialmente, e não por e-mail ou online).

\section{RESULTADOS}

Os dados relativos à descrição sociodemográfica tiveram como objetivo caracterizar a amostra pelo sexo, idade, período do curso, renda familiar, religião e os valores totais de Atitude Religiosa e Bem-Estar Físico e Psicológico. Os dados são apresentados nas Tabelas 1 (Período do curso, sexo, renda familiar e religião) e na Tabela 2 (Idade, Atitude Religiosa e Bem-Estar).

O curso de psicologia nas instituições pesquisadas tem duração de 5 anos ou 10 semestres. A distribuição dos participantes por semestre do curso se deu com um maior número no início do mesmo (semestre $1-\mathrm{N}$ $=32$; semestre $2-\mathrm{N}=42$ e semestre $3-\mathrm{N}=3$ ). No meio do curso participaram 22 estudantes do quinto semestre, 24 do sexto e 14 do sétimo. No final do curso, participaram apenas dois alunos do oitavo semestre, 10 do nono e 11 do décimo.

Quanto ao sexo, houve maior número de participantes do sexo feminino $(82,1 \%)$, sendo que apenas $17,9 \%$ identificaram-se como sendo do sexo masculino.

Boa parte dos estudantes não relatou a renda familiar $(45,8 \% ; \mathrm{N}=77)$. Dentre os que preencheram este item do questionário, houve maior concentração da amostra com renda familiar acima de 10 salários mínimos, o que mostra uma predominância de estudantes de classe média. Apenas 5,5\% dos estudantes afirmam ter renda entre um e dois salários mínimos. A maior parte $(35,2 \%)$ relatou renda familiar entre três e dez salários.

A distribuição dos participantes por religião foi a seguinte: católicos $47,2 \%$, evangélicos $21,4 \%$, espíritas $15,1 \%$, Sem Religião $8,8 \%$. A maior parte dos estudantes $(71,1 \%)$ assume-se como integrante dos grandes grupos religiosos oficialmente descritos pelos órgãos brasileiros de pesquisa (católicos, evangélicos e espíritas). Vale ressaltar que a categoria "Sem Religião" é muito aberta e não implica a ausência de crenças relacionadas à espiritualidade, tais como Deus, vida após a morte, etc. (Novaes, 2004; Pierucci, 2004).

Quanto a distribuição por período do curso, o fato de ter sido maior o número de participantes no início (períodos $1,2,3$ ) influenciou a média que foi igual a $4,33(\mathrm{DP}=2,928)$. 
Tabela 1

Análise descritiva das variáveis categóricas: sexo, renda e religião

\begin{tabular}{|c|c|c|}
\hline VARIÁVEIS & $\mathbf{N}$ & $\%$ \\
\hline \multicolumn{3}{|l|}{ Período } \\
\hline 1 & 32 & 20 \\
\hline 2 & 42 & 26,3 \\
\hline 3 & 3 & 1,9 \\
\hline 5 & 22 & 13,8 \\
\hline 6 & 24 & 15 \\
\hline 7 & 14 & 8,8 \\
\hline 8 & 2 & 1,3 \\
\hline 9 & 10 & 6,3 \\
\hline 10 & 11 & 6,3 \\
\hline \multicolumn{3}{|l|}{ Sexo } \\
\hline Masculino & 30 & 17,9 \\
\hline Feminino & 138 & 82,1 \\
\hline \multicolumn{3}{|l|}{ Renda } \\
\hline $\mathrm{R} \$ 500,00$ a $\mathrm{R} \$ 1.000,00$ & 5 & 5,5 \\
\hline $\mathrm{R} \$ 1.001,00$ a $\mathrm{R} \$ 2.000,00$ & 24 & 26,4 \\
\hline $\mathrm{R} \$ 2.001,00$ a $\mathrm{R} \$ 5.000,00$ & 30 & 33 \\
\hline Acima de R\$5.000,00 & 32 & 35,2 \\
\hline \multicolumn{3}{|l|}{ Religião } \\
\hline Católicos & 75 & 47,2 \\
\hline Evangélicos & 34 & 21,4 \\
\hline Espíritas & 24 & 15,1 \\
\hline Sem Religião & 14 & 8,8 \\
\hline Outros & 12 & 7,5 \\
\hline
\end{tabular}

A Tabela 2 descreve a distribuição dos dados por idade, Atitude Religiosa medida pelo Questionário de Avaliação da Atitude Religiosa (QAAR) e dos valores de Bem-Estar Físico e Psicológico, medido pela Escala de Avaliação do Bem-Estar Físico e Psicológico.
Quanto à idade, observou-se a predominância de estudantes jovens $(M=22,88 ; \mathrm{DP}=7,026)$. A atitude religiosa geral dos participantes apresentou a média de $35,93(\mathrm{DP}=12,55)$, e o Bem-Estar a média de 156,88 $(\mathrm{DP}=35,39)$.

Tabela 2

Análise descritiva das variáveis contínuas: Idade, período do curso, Atitude Religiosa e Bem-Estar

\begin{tabular}{rccccc} 
& $\mathbf{N}$ & Mínimo & Máximo & Média & DP \\
Idade & 129 & 17 & 56 & 22,88 & 7,026 \\
Atitude Religiosa & 160 & 12 & 62 & 35,93 & 12,55 \\
Bem-estar & 163 & 44 & 220 & 156,88 & 35,39 \\
\hline
\end{tabular}

A avaliação das relações entre as questões do QARPSI com o QAAR foi realizada por meio da correlação de Pearson, conforme apresentado na Tabela 3. Os dados mostram que estudantes que se afirmaram como muito religiosos em uma das questões do
QARPSI também apresentaram valores mais altos no índice geral de atitude religiosa obtido pelo QAAR ( $\mathrm{r}$ $=0,786 ; \mathrm{p}<0,001)$ e maior bem-estar $(\mathrm{r}=0,174 ; \mathrm{p}=$ $0,026)$. Observa-se também que quanto mais avançado no curso, mais o aluno percebe as atitudes dos profes- 
sores como desrespeitosas em relação às suas crenças religiosas $(\mathrm{r}=-0,327 ; \mathrm{p}<0,001)$. Uma correlação negativa também foi encontrada entre a idade e a percepção do respeito dos professores às crenças religiosas $(r=-0,219 ; p=0,013)$ e entre a atitude religiosa e a percepção de respeito das crenças pelos professores $(\mathrm{r}=-0,262 ; \mathrm{p}=0,001)$.

Os estudantes que apresentam uma atitude religiosa mais forte, também relataram pensar com maior freqüência nas suas crenças religiosas durante as aulas $(\mathrm{r}=0,687 ; \mathrm{p}<0,001)$ e estudando psicologia fora das salas de aula $(r=0,660 ; p<0,001)$. O bem-estar também apresentou correlação positiva com o pensar nas crenças religiosas durante as aulas $(\mathrm{r}=0,239 ; \mathrm{p}=$ $0,002)$ e fora das salas de aula $(r=0,248 ; p=0,002)$.

$\mathrm{O}$ apoio da família para cursar psicologia não apresentou relação significativa com nenhuma das questões estudadas. Entretanto, foi observada uma correlação positiva entre a atitude religiosa e o apoio da comunidade religiosa para a escolha por cursar psicologia.

Também foi observada correlação positiva entre a atitude religiosa e a percepção do estudante de que há conflito entre as suas crenças religiosas e algumas abordagem de psicologia $(r=0,536 ; p<0,001)$. Foi solicitado aos participantes que listassem as abordagens que eles percebiam como estando em maior conflito com suas crenças religiosas. Dos 168 participantes, apenas 54 apontaram alguma abordagem. Isso pode ter ocorrido devido ao fato de grande parte dos participantes estarem iniciando o curso e ainda não terem familiaridade com as abordagens. Dentre os participantes que responderam, todavia, $61 \%$ apontaram a Psicanálise como abordagem em maior conflito com suas crenças religiosas, $14,8 \%$ apontaram o Behaviorismo e $9,3 \%$ a Gestalt.

Tabela 3

Correlação de Pearson entre questões respondidas pelos participantes acerca da sua religiosidade e suas relações com o curso de psicologia e o período do curso, idade, atitude religiosa e bem-estar físico e psicológico

\begin{tabular}{rrrrr}
\multicolumn{1}{r}{ Variável } & Período & \multicolumn{1}{c}{ Idade } & $\begin{array}{c}\text { Atitude } \\
\text { Religiosa }\end{array}$ & Bem-Estar \\
\hline Q-Relig & 0,090 & 0,065 & $\mathbf{0 , 7 8 6 * *}$ & $\mathbf{0 , 1 7 4 *}$ \\
R-Prof & $\mathbf{- 0 , 3 2 7 * *}$ & $\mathbf{- 0 , 2 1 9 *}$ & $\mathbf{- 0 , 2 6 2 * *}$ & $-0,096$ \\
CRE-Sala & $-0,068$ & $-0,152$ & $\mathbf{0 , 6 8 7 * *}$ & $\mathbf{0 , 2 3 9 * *}$ \\
CRE-Fora & $-0,002$ & $\mathbf{0 , 2 6 6 * *}$ & $\mathbf{0 , 6 6 0 * *}$ & $\mathbf{0 , 2 4 8 * *}$ \\
AB-Confl & 0,020 & 0,092 & $\mathbf{0 , 5 3 6 * *}$ & 0,070 \\
PSI-ABAND & $-0,087$ & $-0,076$ & 0,119 & $-0,104$ \\
Apoio F & 0,082 & 0,053 & 0,097 & 0,090 \\
Apoio CR & 0,067 & 0,134 & $\mathbf{0 , 4 3 4 * *}$ & 0,030 \\
\hline
\end{tabular}

* Correlação significativa a 0,01 (two-tailed)

** Correlação significativa a 0,05 (two-tailed)

NOTA: Q-RELIG = 0 quanto você é religioso?; R-PROF = Os professores de psicologia respeitam suas crenças religiosas?; CRE-SALA = Com que frequência, durante as aulas de psicologia, você se lembra de suas crenças religiosas?; CRE-FORA = Com que frequência, ao estudar psicologia fora das salas de aula, você se lembra de suas crenças religiosas?; AB-CONFL= Há conflito entre as suas crenças religiosas e algumas (s) abordagem (s) de psicologia?; PSI-AB = A formação em psicologia, como um todo, faz com que as pessoas abandonem suas crenças religiosas?; APOIO F = A sua família apóia a sua escolha por cursar psicologia?; APOIO C R = As pessoas da sua comunidade religiosa apóiam a sua escolha por cursar psicologia?

A Tabela 4 mostra as correlações entre as próprias questões do QARPSI (Q-Relig, R-Prof, CRE-Sala, CRE-Fora, AB-Confl, PSI-ABORD, Apoio F e Apoio $\mathrm{CR})$. Observou-se que quanto mais os estudantes afirmam-se como religiosos, mais percebem desrespeito por parte dos professores de psicologia por suas crenças religiosas $(r=-0,212 ; p=0,006)$. Esta correlação confirma a que foi apresentada na Tabela 1 , entre atitude religiosa (medida pelo QAAR) e a percepção de desrespeito dos professores. Valores mais altos no au- torrelato do quanto se é religioso mostraram correlação positiva com o pensar nas crenças dentro $(r=0,606 ; p$ $<0,001)$ e fora de sala de aula $(\mathrm{r}=0,546 ; \mathrm{p}<0,001)$. Observou-se também uma coerência dos dados da Tabela 3 e da Tabela 4, na correlação positiva entre o afirmar-se como muito religioso (questão do QARPSI) e perceber conflito com algumas abordagens de psicologia $(r=0,430 ; p<0,001)$. Do mesmo modo, estudantes que se afirmaram como muito religiosos também tenderam a considerar que sua escolha pelo curso de 
psicologia foi mais apoiada pela comunidade religiosa da qual faz parte $(\mathrm{r}=0,298 ; \mathrm{p}<0,001)$.

Uma correlação negativa foi observada entre a percepção de respeito por parte dos professores de psicologia em relação às crenças religiosas dos estudantes e o conflito entre tais crenças e algumas abordagens de psicologia $(r=-0,354)$. Essa correlação aponta que quanto mais respeitosos os professores são com as crenças, menos conflito é percebido entre estas e algumas abordagem de psicologia.
Também foi observada uma correlação positiva entre a percepção de conflito com as abordagens e a afirmação de que as pessoas podem abandonar suas crenças religiosas por estudarem psicologia $(r=0,323$; $\mathrm{p}<0,001)$.

Por último, é interessante observar que os estudantes que se consideram apoiados pela família para cursar psicologia, também se sentem apoiados pela sua comunidade religiosa $(r=0,353 ; p<0,001)$.

Tabela 4

Correlação de Pearson entre as questões respondidas pelos participantes acerca da sua religiosidade e suas relações com o curso de psicologia

\begin{tabular}{|c|c|c|c|c|c|c|c|c|}
\hline Variável & Q-Relig & R-Prof & CRE-Sala & CRE-Fora & AB-Confl & $\begin{array}{c}\text { PSI- } \\
\text { ABAND }\end{array}$ & Apoio F & $\begin{array}{c}\text { Apoio } \\
\text { CR }\end{array}$ \\
\hline Q-Relig & 1 & $-0,212 * *$ & $0,606 * *$ & $0,546 * *$ & $0,430 * *$ & 0,26 & 0,049 & $0,298 * *$ \\
\hline R-Prof & & 1 & $-0,131$ & $-0,096$ & $-354 * *$ & $-0,124$ & $-0,081$ & $-0,017$ \\
\hline CRE-Sala & & & 1 & $\mathbf{0 , 8 0 4 * *}$ & $0,463 * *$ & 0,141 & 0,077 & $0,292 * *$ \\
\hline CRE-Fora & & & & 1 & $0,421 * *$ & $0,179 *$ & 0,031 & $0,286^{* *}$ \\
\hline AB-Confl & & & & & 1 & $0,323 * *$ & 0,015 & $-0,069$ \\
\hline PSI-ABAND & & & & & & 1 & $-0,038$ & $-0,096$ \\
\hline Apoio F & & & & & & & 1 & $\mathbf{0 , 3 5 3} * *$ \\
\hline Apoio CR & & & & & & & & 1 \\
\hline
\end{tabular}

* Correlação significativa a 0,01 (two-tailed)

** Correlação significativa a 0,05 (two-tailed)

NOTA: Q-RELIG = 0 quanto você é religioso?; R-PROF = Os professores de psicologia respeitam suas crenças religiosas?; CRE-SALA = Com que frequência, durante as aulas de psicologia, você se lembra de suas crenças religiosas?; CRE-FORA = Com que frequência, ao estudar psicologia fora das salas de aula, você se lembra de suas crenças religiosas?; $A B-C O N F L=$ Há conflito entre as suas crenças religiosas e algumas (s) abordagem (s) de psicologia?; PSI-ABNAD = A formação em psicologia, como um todo, faz com que as pessoas abandonem suas crenças religiosas?; APOIO F = A sua família apóia a sua escolha por cursar psicologia?; APOIO C R = As pessoas da sua comunidade religiosa apóiam a sua escolha por cursar psicologia?

\section{DISCUSSÃO}

O presente trabalho teve como objetivos verificar as relações entre o bem-estar e a religiosidade; verificar se há conflito entre o curso de psicologia e as crenças religiosas dos estudantes, e; avaliar a percepção dos alunos das atitudes dos professores frente às suas crenças.

Examinando a relação entre religiosidade e bem-estar, verificou-se que os estudantes que se afirmaram como mais religiosos, também apresentaram índices mais altos de bem-estar físico e psicológico. Esses dados evidenciam a religiosidade como possível fator de proteção (Gastaud et al., 2006; Stroppa \& Moreira-Almeida, 2008). Apesar das análises correlacionais não oferecerem condições para afirmação de relações causais, chama a atenção o fato de que tais resultados confirmem o que os diferentes estudos têm apontado.
Isso, considerando que relatos de bem-estar positivo são sinais legítimos de saúde, no seu sentido biopsicossocial (Passareli \& Silva, 2007).

Os resultados também apontam que há tensão entre as crenças religiosas dos estudantes e o curso de psicologia. Contudo, a tensão parece estar mais diretamente relacionada a abordagens específicas e não necessariamente ao curso de modo geral. A Psicanálise foi a abordagem mais citada, seguida pelo Behaviorismo. Ambas as abordagens, apesar de suas diferentes epistemologias, são representantes do modelo científico da modernidade e se desenvolveram sob influência de uma cultura de oposição às tradições religiosas que antes mediavam as relações das pessoas com a realidade (Oliveira, Pires, \& Vieira, 2009). A histórica oposição da Psicanálise à religião e o espírito científico pragmático do Behaviorismo e sua concepção das instituições religiosas como agências contro- 
ladoras do comportamento que utilizam a coerção como uma das principais formas de controle (ameaça de punição, por exemplo), podem estar influenciando as percepções dos estudantes quanto ao conflito da formação com suas crenças religiosas (Freud. 1927/ 1969; Skinner, 1953).

Deve-se destacar, entretanto, que os professores são mediadores importantes na relação dos alunos com a formação. Sendo os professores, em grande parte, representantes de abordagens da psicologia, é necessário perguntar se os alunos fazem uma separação clara entre os posicionamentos pessoais dos professores e as abordagens que eles representam. Esta é uma pergunta importante, considerando que os estudantes perceberam a existência de conflito de suas crenças tanto com as abordagens como com professores. Apesar de não ter sido observada uma relação direta entre o respeito dos professores às crenças e o bem-estar, observam-se indícios de que o desrespeito ocorre. Se há evidências de que as crenças religiosas estão diretamente relacionadas ao bem-estar dos estudantes e se os professores, realizando uma mediação negativa da relação com a religião, contribuem para o conflito com as mesmas, então é possível considerar que seus posicionamentos podem afetar negativamente o bem-estar dessas pessoas. Considerando a religiosidade dos alunos como possível fator de proteção, pode-se inferir que esse tipo de mediação realizada pelos professores seja prejudicial à saúde dos alunos (Gastaud et al., 2006; Stroppa \& Moreira-Almeida, 2008; Volcan, Sousa, \& Horta, 2003).

Os dados mostraram relações significativas entre o ser muito religioso, estar mais avançado no curso e ter maior percepção de que os professores de psicologia não respeitam as crenças religiosas dos seus alunos. Tais resultados oferecem contribuição significativa, por alertar aos professores sobre a importância de repensarem o modo como revelam os seus posicionamentos frente a conteúdos que são sensíveis, e que podem deixar os estudantes psicologicamente mais vulneráveis ao desenvolvimento de transtornos mentais (Volcan et al., 2003).

$\mathrm{O}$ apoio familiar e o apoio da comunidade religiosa também parecem importantes, mostrando ligações entre si. É interessante notar, entretanto, a importância social dos grupos religiosos para os estudantes que deles fazem parte. $\mathrm{O}$ apoio dos grupos religiosos para que o estudante se forme em psicologia pode ser um fator que contribui para a manutenção do bem-estar, talvez por contribuir com a significação do exercício da profissão de psicólogo e pelo status que pessoas com esta formação possuem nas comunidades religiosas. Em estudos posteriores, sugere-se investigar as percepções dos estudantes que se sentem apoiados socialmente pelo seu grupo religioso, acerca de possíveis relações com o futuro exercício da profissão neste contexto. Pode ser investigada a hipótese de que os próprios grupos religiosos possam estar incentivando seus jovens a estudarem psicologia, por considerarem que os psicólogos podem contribuir com os serviços que prestam à sociedade e ao seu público interno.

As principais contribuições deste trabalho são: 1) A confirmação da relação entre religiosidade e bem-estar, inclusive em estudantes de psicologia no contexto da formação; 2) A identificação de tensões entre a religiosidade e o curso, e; 3) A apresentação de dados que alertam aos professores quanto aos possíveis desdobramentos de seus posicionamentos frente a crenças que podem ser importantes para a manutenção da saúde dos estudantes. Mesmo que se admita que o impacto sobre crenças pessoais e religiosas faça parte do processo de formação, é importante considerar os cuidados necessários para que os estudantes se desenvolvam do modo mais saudável possível.

Cabe uma reflexão acerca da faixa etária predominante no curso de psicologia investigado. Se for tomada como referência a abordagem da resiliência como processo de desenvolvimento no qual os recursos vão se tornando mais fortes com o vivenciar das experiências, pode ser razoável considerar como mais seguro iniciar o curso de psicologia em idade mais madura do que a observada (Fontes, 2010). É difícil, todavia, estabelecer uma idade ideal para iniciar o curso, porque há diferenças individuais que questionam a idade como fator principal para determinar a vulnerabilidade ou não para o enfrentamento de situações desafiadoras. Alguns indivíduos mais jovens podem mostrar-se mais resilientes diante das dificuldades da formação, do que outros biologicamente mais maduros. Este trabalho, então, pretende também contribuir chamando a atenção para o fato de que, se não há como determinar uma idade ideal para iniciar o curso de psicologia, pode ser possível a realização de trabalhos preventivos, visando o desenvolvimento de habilidades cognitivas e comportamentais de jovens estudantes ao início do curso. Estudantes psicologicamente mais saudáveis podem se tornar também, no futuro, profissionais mais preparados para cumprir uma de suas principais missões como psicólogos: a promoção da saúde humana.

O presente estudo não investigou se os estudantes se tornam menos religiosos ao longo do curso, o que 
poderia ser investigado em estudos posteriores. Investigaram-se apenas as percepções que os estudantes têm acerca da interação entre a sua religiosidade e fatores presentes na formação acadêmica. Os resultados mostraram que mesmo percebendo conflitos entre suas crenças e fatores presentes no processo de formação, o bem-estar não é afetado negativamente. Tal resultado, entretanto, pode estar revelando apenas as percepções dos estudantes que não se tornaram menos religiosos ao longo do curso. É interessante notar que quanto mais avançados no curso, maior é a percepção dos estudantes de que as crenças religiosas estão em conflito com a formação em psicologia. A percepção desse conflito parece ganhar força na medida em que o contato com as abordagens e com professores de várias delas ao longo curso vão ocorrendo. Alguns estudantes podem desenvolver estratégias para protegerem-se das ameaças às suas crenças, enquanto outros possivelmente passam por um processo de relativização e enfraquecimento das mesmas. Considerando a relevância das crenças religiosas e suas interações com o bem-estar, uma investigação cuidadosa acerca de tais processos é recomendada para novos trabalhos.

\section{REFERÊNCIAS}

Beck, J. S. (1997). Terapia Cognitiva: teoria e prática. Porto Alegre: Artmed.

Bouteyre, E., Maurel, M., \& Bernaud J. L. (2007). Daily hassles and depressive symptoms among first year psychology students in France: the role of coping and social support. Stress and Health, 23(2), 93-99.

Cavestro, J. M. \& Rocha, F. L. (2006). Prevalência de depressão entre estudantes universitários. Jornal Brasileiro de Psiquiatria, 55(4): 264-267.

Cerchiari, E. A. N. (2004). Saúde mental e qualidade de vida em estudantes universitários. Tese de doutorado. Faculdade de Ciências Médicas da Universidade Estadual de Campinas, Campinas, São Paulo, SP, Brasil.

Costa, C. C.; Bastiani, M.; Geyer, J. G.; Calvetti, P. U.; Muller, M. C., \& Moraes, M. L. A (2008). Qualidade de vida e bem-estar espiritual em universitários de psicologia. Psicologia em Estudo, Maringá, v. 13, n. 2, p. 249-255.

Diener, E. (1984). Subjective well-being. Psychological Bulletin, $95,542-575$

Falcone, E. (2001). Psicoterapia cognitiva. In Rangé, B. (Ed.). Psicoterapias cognitivo-comportamentais: um diálogo com a psiquiatria. Porto Alegre: Artmed.

Fleck, M. P. A.; Borges, Z. N.; Bolognesi, G., \& Rocha, N. S. (2003). Desenvolvimento do WHOQOL, módulo espiritualidade, religiosidade e crenças pessoais. Rev Saúde Pública; 37(4):44655 .
Fleck, M.P.A. et al. (1999). Desenvolvimento da versão em português do instrumento de avaliação de qualidade de vida da OMS (WHOQOL-100). Revista Brasileira de Psiquiatria, 21(1), 1928.

Fontes, A. P. (2010). Resiliência, segundo o paradigma do desenvolvimento ao longo da vida (life-span). Revista Kairós, Caderno Temático 7, pp. 8-20.

Freud, S. (1907). Atos obsessivos e práticas religiosas. In J. Salomão (Ed.), Edição Standard Brasileira das Obras Psicológicas Completas de Sigmund Freud (V. IX, pp. 105-117). Rio de Janeiro: Imago. (Trabalho originalmente publicado em 1907.)

Freud, S. (1969). O futuro de uma ilusão. In J. Salomão (Ed.), Edição standard brasileira das obras psicológicas completas de Sigmund Freud (Vol. XI, pp. 13-71). Rio de Janeiro: Imago. (Trabalho original publicado em 1927.)

Gastaud, M. B.; Souza, L. D. M.; Braga, L.; Horta, C. L.; Oliveira, F. M.; Sousa, P. L. R., \& Silva, R. A. (2006). Bem-estar espiritual e transtornos psiquiátricos menores em estudantes de psicologia: estudo transversal. Revista Psiquiatria; 28(1):12-18.

Giddens, A. (1991). As conseqüências da modernidade. São Paulo: Editora da UNESP.

Jarros, R. B.; Dias, H. Z. J.; Müller, M. C., \& Sousa, P. L. R. (2008). Estudo bibliométrico da produção brasileira na interface da psicologia com espiritualidade-religiosidade. PSIC - Revista de Psicologia da Vetor Editora, v. 9, n. 2, p. 251-258.

Jessor, R.; Van Den Bos, J.; Vanderryn, J.; Costa, F. M., \& Turbin, M. S. (1995). Protective factors in adolescent problem behavior: moderator effects and developmental change. Developmental Psychology, v. 31, n. 6, 923-933.

Koenig, H. G. (2007). Religião, espiritualidade e psiquiatria: uma nova era na atenção à saúde mental. Revista de Psiquiatria Clíni$c a$, vol. 34, suppl. 1, pp. 5-7.

Lazarus, R.S. \& Folkman, S. (1984). Stress, appraisal and coping, New York: Springer.

Novaes, R. (2004). Os jovens "sem religião": ventos secularizantes, "espíritos de época" e novos sincretismos. Notas preliminares. Estudos Avançados, 18(52), 321-330.

Oliveira, P.; Torres, A. R. R., \& Albuquerque, E. S. Análise do bem-estar psicossocial de aposentados de Goiânia. Psicologia em Estudo, v. 14, n. 4, pp. 749-757.

Oliveira, C. I; Pires, A. C., \& Vieira, T. M. (2009). A terapia cognitiva de Aaron Beck como reflexividade na alta modernidade: uma sociologia do conhecimento. Psicologia: Teoria e Pesquisa, 24(4), 637-645.

Panzini, R. G.; Rocha, N. S.; Bandeira, D. R., \& Fleck, M. P. A. (2007). Qualidade de vida e espiritualidade. Rev. Psiq. Clín. 34, supl. 1, p. 105-115.

Panzini, R.G. \& Bandeira, D. R.(2005). Escala de Coping ReligiosoEspiritual (Escala CRE): elaboração e validação de construto. Psicologia em Estudo 10(3), 507-16.

Panzini, R. G.(2004). Escala de coping religioso-espiritual (Escala CRE): tradução, adaptação e validação da escala RCOPE, 
abordando relações com saúde e qualidade de vida. Dissertação de mestrado não publicada. Universidade Federal do Rio Grande do Sul, Porto Alegre, Rio Grande do Sul, Brasil.

Pargament, K. I. (1997). The psychology of religion and coping: Theory, research, practice. New York: Guilford Press.

Passareli, P. M. \& Silva, J. A. (2007). Psicologia positiva e o estudo do bem-estar subjetivo. Estudos de Psicologia, 24(4), pp. 513517.

Paulo Junior, W. \& Zanini, D. S. (2011). Estratégias de Coping de Pacientes Oncológicos em Tratamento Radioterápico. Psicologia: Teoria e Pesquisa, Vol. 27, n. 4, pp. 491-497.

Pesce, R. P.; Assis, S. G.; Santos, N., \& Oliveira, R. V. C. (2004). Risco e proteção: em busca de um equilíbrio promotor de resiliência. Psicologia: Teoria e Pesquisa, Vol. 20, n. 2, pp. 135143.

Pierucci, A. F. (2004). "Bye bye, Brasil": o declínio das religiões tradicionais no Censo 2000. Estud. av., vol. 18, n. 52, 17-28.

Pinheiro, D. P. N. (2004). A resiliência em discussão. Psicologia em Estudo, v. 9, n. 1, pp. 67-75.

Sakae, T. M.; Padão, D. L., \& Jornada, L. K. (2010). Sintomas depressivos em estudantes da área da saúde em uma Universidade no Sul de Santa Catarina - UNISUL. Revista da Associação Médica do Rio Grande do Sul (AMRIGS), Porto Alegre, 54(1), 38-43.

Seybold, K. S. \& Hill, P. C. (2001). The Role of Religion and Spirituality in Mental and Physical Health. Current Directions in Psychological Science, 10(1), 20-24.

Siqueira, M. M. M. \& Padovam, V. A. R. (2008). Bases teóricas de bem-estar subjetivo, bem-estar psicológico e bem-estar no trabalho. Psicologia: Teoria e Pesquisa, v. 24, n. 2, pp. 201-209.

Shimmack, U. (2008). The Structure of subjective well-being. In Eid, M. \& Larsen, R. J (Eds.) The science of subjective wellbeing (pp. 97-123). New York: Guilford Press.
Skinner, B.F. (1953). Ciência e comportamento humano. São Paulo: Martins Fontes.

Snyder, C. R. \& Lopez, S. J. (2009). Psicologia positiva: uma abordagem científica e prática das qualidades humanas. Porto Alegre: Artmed.

Steel, P.; Schmidt, J., \& Shultz, J. (2008). Redefining the relationship between personality and subjective well-being. Psychological Bulletin, 134(1), 138-161.

Stroppa, A. \& Moreira-Almeida, A. (2008). Religiosidade e Saúde. Salgado, M. I. \& Freire, G. (Eds.) Saúde e Espiritualidade: Uma nova visão da medicina. (pp. 427-443).

Teixeira, Fonseca , \& Máximo. (2002). Inventário SF36: avaliação da qualidade de vida dos alunos do curso de Psicologia do Centro UNISAL - U.E. de Lorena (SP). PSIC - Revista de Psicologia da Vetor Editora, Vol. 3, n. 1, pp. 16-27.

Volcan, S. M. A.; Sousa, P. L. R., \& Horta, B. L. (2003). Relação entre bem-estar espiritual e transtornos psiquiátricos menores: estudo transversal. Rev. Saúde Pública, 37(4), pp. 440-445.

Yoon, J. \& Lau, A. S. (2008). Maladaptive perfectionism and depressive symptoms among Asian American college students: contributions of interdependence and parental relations. Cultural Diversity \& Ethnic Minority Psychology, 14(2), 92-101. 